\title{
Introducing students to single photon detection with a reverse-biased LED in avalanche mode
}

\author{
Lowell I. McCann ${ }^{1}$ \\ ${ }^{1}$ University of Wisconsin - River Falls, Physics Department, \\ 410 S. $3^{\text {rd }}$ St., River Falls, WI 54022
}

\begin{abstract}
An inexpensive experiment using a reverse-biased LED as an avalanche photodiode is described. The experiment is rich in physics topics and experimental techniques, allowing students to explore the statistics of random events, basic discriminator circuits, and the behavior of avalanche photodiodes.
\end{abstract}

PACS: $01.50 . \mathrm{Pa}$, 85.60.Dw, 42.50.Ar, 84.30.Sk

\section{INTRODUCTION}

Over the past two decades, the popularity of single photon experiments in undergraduate laboratory courses has increased dramatically. This is a result of technological advances and the effort of a dedicated group of researchers who have developed experiments investigating basic principles of quantum mechanics that are suitable for undergraduates to perform [1-7].

The detectors used in these experiments are typically single photon counting modules (SPCMs) based around avalanche photodiodes, with a signal conditioning circuit to produce an easily read voltage pulse when a photon is detected. While the cost of SPCMs for educational use has been reduced [8], they are still substantial investments for many physics departments. In addition, the sensitivity of the SPCMs require that students handle them carefully in order to not damage them, so students may not be allowed to 'play' with them to see how they work and to determine their limitations. This can lead to students viewing the SPCM as a 'black box' that they don't understand.

Students are usually introduced to the statistics of random events using radioactive decay [9-11], even though the arrival of photons at a detector can obey the same statistical relationships. The results students get from a simple experiment using Cs-137 and a Geiger-Müller tube typically agree beautifully with theoretical predictions. However, in these experiments, there are not often a lot of parameters under the control of the experimenter. One important parameter that is typically fixed is the 'dead time' of the detector - the time during which the detector is recovering from an event during which it is unable to sense another event.
In this paper I describe a pedagogically rich experiment suitable for sophomore through senior undergraduate students where a very inexpensive light emitting diode (LED) is used as an insensitive avalanche photodiode with which students can examine counting statistics, basic discriminator circuits, and the properties of semiconductors -with many parameters for the students to explore. The basic physics of an avalanche photodiode and the statistics describing the arrival of photons will be discussed first, followed by a description of the experimental design, typical data collected, and a discussion of other investigations that could be made.

\section{A. Geiger-mode Avalanche Photodiodes}

The basic physics of an avalanche photodiode is very straight-forward $[12,13]$. A $p-n$ junction is formed within a semiconductor material, and the junction is reverse-biased. At low bias voltages, no current will flow through the junction, because charge carriers do not have enough energy to overcome the potential energy barrier created at the depletion region of the junction. At sufficiently high reverse bias voltages, if a photon is absorbed in the junction and creates an electron-hole pair, the large electric field in the junction will cause the pair to separate before they have a chance to recombine. As the excited electron accelerates in the electric field, it gains kinetic energy. If the electron interacts with an atom within the junction, that kinetic energy can be converted into the creation of another electon-hole pair. Now there are two electrons that are accelerated by the field, and the avalanche process continues, so that a single photon can produce a small but measureable pulse of current. Figure 1 illustrates this process. This mode of operation is called the Geiger mode, in reference to the great similarity with the behavior of Geiger-Müller tubes. 


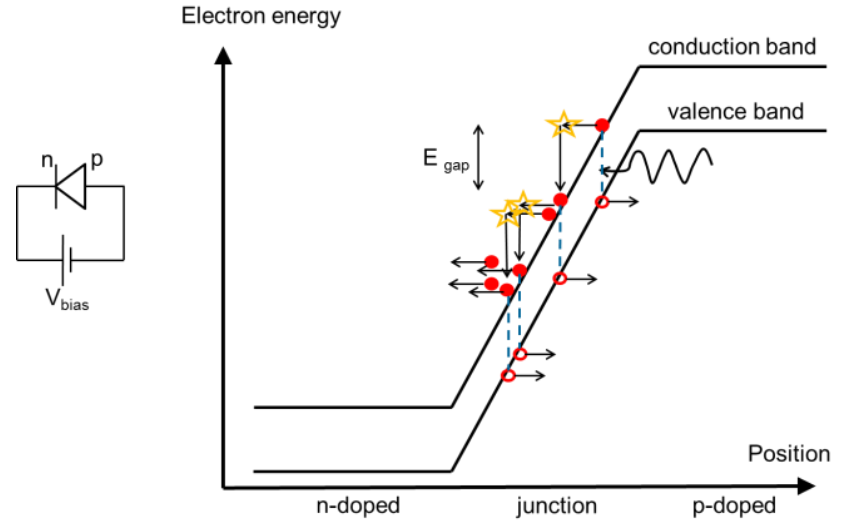

FIG 1. Electron energy level diagram across a reverse biased p-n junction. A photon excites an electron-hole (solid circle and empty circle, respectively) pair which are accelerated by the electric field. Collisions (stars) create additional electron hole pairs, leading to an avalanche. Inset: a schematic of a reverse-biased diode.

Photon interactions are not the only way to cause an avalanche. Thermal energy can excite electrons to the conduction band as well, and commercial avalanche photodiodes are often cooled below room temperature to reduce the number of 'dark counts' - avalanches that are not initiated by photons. This dark count rate is dependent upon the construction of the photodiode and the bias voltage as well.

The avalanche does not continue indefinitely unless the bias voltage is too large or the quenching resistor in series with the photodiode is too small [14]. The time for the pulse to be quenched determines the 'dead time' of the detector - the time during which it is impossible to detect the arrival of another photon. In the experiment described here, the dead time is determined by factors under the control of the student.

While the avalanche is occurring, current is flowing through the junction and, via Joule heating, causing the temperature of the junction to increase. This increased temperature enhances the probability that after the avalanche stops, another one will begin due to thermal excitation. This is one of the main causes of afterpulsing. Afterpulsing is the phenomenon by which one avalanche begets a second (or more) avalanche. These avalanches are indistinguishable from an avalanche caused by another photon, and leads to an over count of the number of photons. Much effort has been made to reduce afterpulsing in avalanche photodiode circuits $[15,16]$.

\section{B. Single Photon Counting Statistics}

Under the proper conditions [17], the arrival of photons at a detector should occur at random, uncorrelated times. This means that photon detection should exhibit the same statistical behavior as radioactive decay [18]. A Poisson distribution is expected to describe the number of events counted in a given interval of time:

$$
p(j, a)=\frac{a^{j} e^{-a}}{j !}
$$

where $p$ is the probability of measuring $j$ events in a specified interval if $a$ is the average number of events found during that interval [19]. An exponential distribution is expected to describe the time intervals between subsequent photon arrivals:

$$
p(t) d t=r e^{-r t} d t
$$

where $p(t) d t$ is the probability that one random event is followed by another event during a window of time $d t$ and at a time $t$ after the previous event, with $r$ being the average rate of events. [20]

It is common to use radioactive decay measurements to examine both of those distributions. In fact, the measurement of the distribution of time between events is an excellent way to quickly determine the dead time of a detection system, because the dead time leads to predictable deviations from the expected $p(t)$ at short time intervals.

\section{EXPERIMENTAL PROCEDURE}

In this experiment, a Gallium Phosphide [21] LED (Purdy Electronics Corp., AND114R) is used as a very inexpensive photodiode. In part because it was manufactured to produce rather than detect light, the LED has a very low quantum efficiency. While this makes it a poor choice as a detector, it is convenient to use with students in a well-lit classroom, and its cost means there is no concern if the LED gets damaged. Both factors make this a good choice when encouraging students to explore. We have so far found only this and the AND113 LEDs to work as Geiger mode photodiodes at $\mathrm{V}_{\text {bias }}<30 \mathrm{~V}$.

A simple passively-quenched, reverse-biased LED circuit is shown on the left side of Fig. 2. When no current is flowing, no voltage drop occurs across the quenching resistor in series with the LED, but when an avalanche occurs the voltage on the non-grounded side will increase, typically in the range of 100's of $\mathrm{mV}$. To detect these voltage pulses with a computer it is convenient to add a 


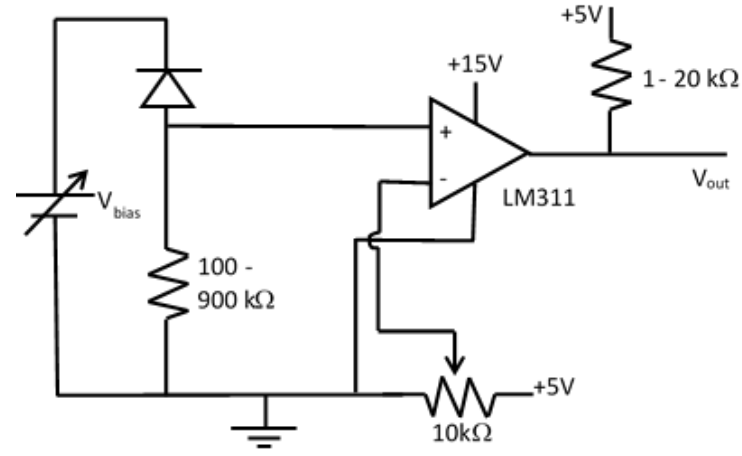

FIG 2. Circuit diagram for reverse-biasing an LED and using an op-amp comparator (LM311) to produce a $5 \mathrm{~V}$ output pulse. $\mathrm{V}_{\text {bias }}$ is typically around $24 \mathrm{~V}$ for the AND114R LEDs.

discriminator to the output. Here, an inexpensive LM311 op-amp comparator is used for this purpose. The output will remain at ground until a voltage pulse at the noninverting input (+) of the op-amp rises above the voltage at the inverting input (-). The 'pull-up' resistor on the output sets the amplitude of the output pulses to $5 \mathrm{~V}$. The voltage at the inverting input is controlled by the potentiometer between the inverting input and the $5 \mathrm{~V}$ supply.

This basic discriminator circuit is highly useful in getting students to understand the triggering on an oscilloscope. By monitoring the voltage at the two inputs and the output of the op-amp, students can visually see that $5 \mathrm{~V}$ output pulses only occur when the original pulses cross the voltage threshold set by the potentiometer. This forces students to confront the idea that they are discarding some events based on the discriminator level.

Finally, the TTL-level pulses produced can be detected by any number of devices. We have used oscilloscopes, LabView DAQs, Vernier LabQuests, and TeachSpin's Pulse Counter/Interval Timer. With these devices, tests of the statistical properties of the arrival of photons at the detector are possible.

\section{RESULTS}

Figure 3 illustrates typical distributions of the number of counts per interval compared to Poisson and Gaussian distributions with the same mean and standard deviation as the data. The data (using ambient room light as the source) is reasonably well described by a Poisson distribution for all three intervals. The noticeable discrepancy is due mostly to afterpulsing, which causes the distribution's variance to not be equal to its mean as expected for Poisson distributions. As expected, the Gaussian matches poorly for small average numbers of counts, but agrees very well
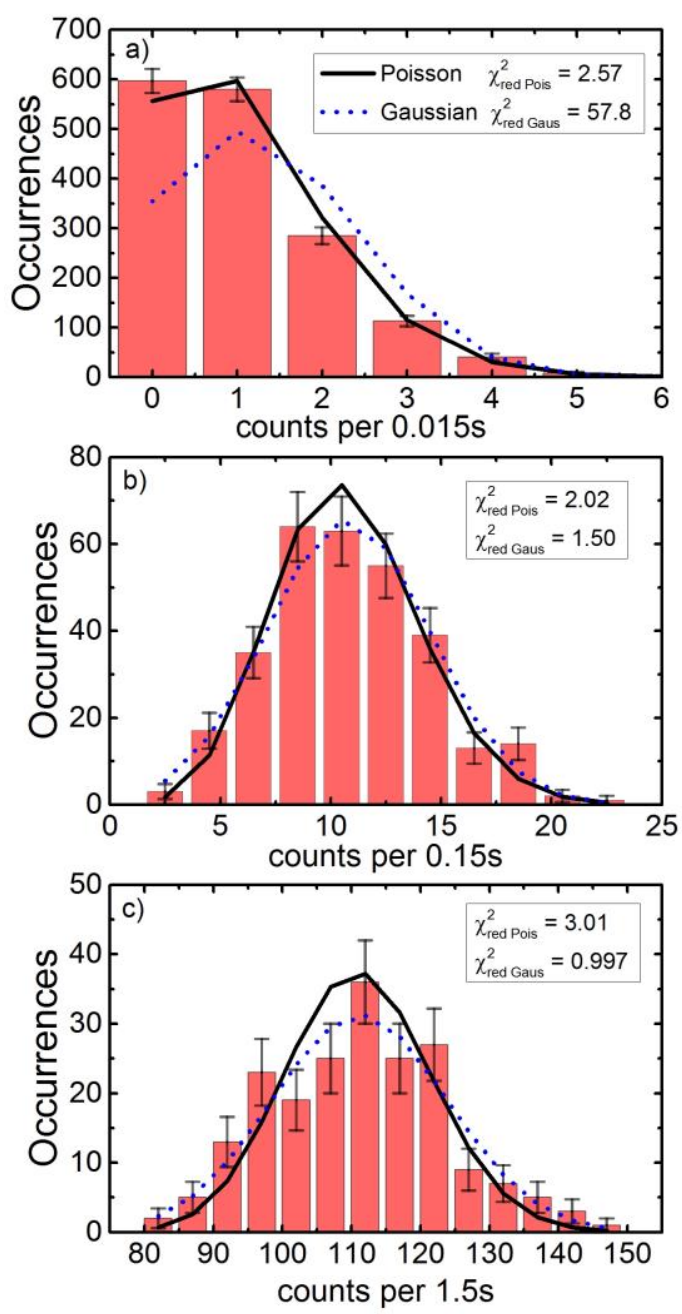

FIG 3. Histograms of the number of counts per interval for intervals of a) $0.015 \mathrm{~s}, \mathrm{~b}) 0.15 \mathrm{~s}$, and c) $1.5 \mathrm{~s}$. Poisson (solid) and Gaussian (dashed) distributions with the same mean and standard deviations as the data are shown as well. The error bars represent the square root of the values.

when the average increases and the distribution becomes symmetric.

Figure 4 shows typical data of the time between pulses. The expected exponential distribution is broken at very short time intervals by a large spike as a result of the afterpulsing. Those correlated pulses are produced at very short intervals after a 'real' pulse and are easily distinguished in this plot. The dead time of this detection system can be controlled by the choice of the quenching resistor in series with the LED as seen in Fig 5.

\section{CONCLUSIONS}

This experimental system provides another inexpensive means by which students can investigate the statistics of 


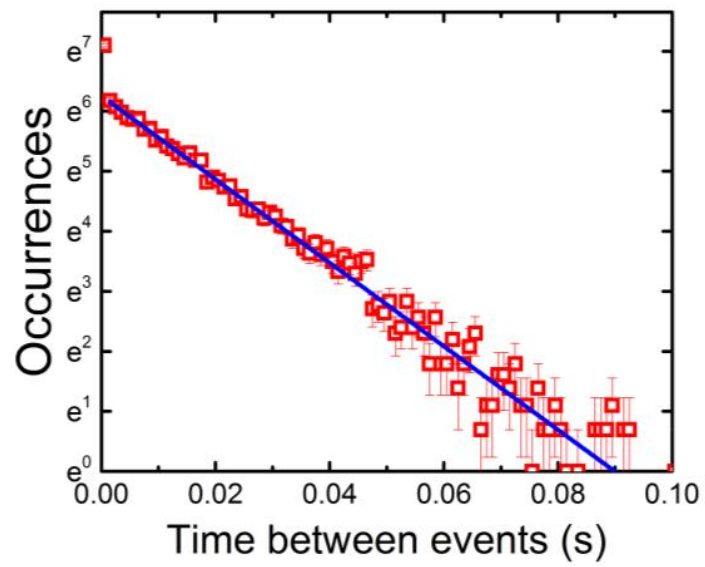

FIG 4. Histogram of the time between events (pulses) showing the expected exponential behavior after a large peak due to afterpulsing. The raw average count rate was $74 \mathrm{cts} / \mathrm{s}$, and the quenching resistor was $900 \mathrm{k} \Omega$. The fit line shown (blue) is: Occ. $=(521.1 \pm 9.2) \mathrm{e}^{-\mathrm{t} /(0.01440 .0002 s)}$, which yields a rate (not including afterpulses or dead time effects) of $69 \pm 1 \mathrm{cts} / \mathrm{s}$. The inset of Fig. 5 includes a close up view of the same data at short time intervals.

random events, learn the operation of avalanche photodiodes, and learn the basics of discriminator circuits.

Students can also investigate: the temperature dependence of the dark count rate, the spectral response, coherent light sources, the impact of the plastic coating over the $\mathrm{p}-\mathrm{n}$ junction, pulse height analysis of the original pulses from the LED, corrections to the data to remove the impact of afterpulsing, the distribution of dead times, as well as designing and testing active-quenching circuits.

[1] D. Dehlinger, M. W. Mitchell, Am. J. Phys. 70(9), 903910 (2002).

[2] E.J. Galvez, et al., Am. J. Phys. 73 (2), 127-140 (2005).

[3] J. J. Thorn, et al., Am. J. Phys. 72, 1210 (2004).

[4] B. J. Pearson and D. P. Jackson, Am. J. Phys. 78, 471 (2010).

[5] E. Dederick and M. Beck, Am. J. Phys. 82, 962 (2014).

[6] M. Beck, Quantum Mechanics: Theory and Experiment, (Oxford University Press, Oxford, NY, 2012) p. 435-436.

[7] E. J. Galvez, Am. J. Phys. 82, 1018 (2014).

[8] ALPhA's Single Photon Detector Initiative, www.advlab.org/spqm.html

[9] E. Mathieson and T. J. Harris, Am. J. Phys. 38, 1261 (1970).

[10] E. J. Gucker, F. K. Milano, and R. V. Mancuso, Am. J. Phys. 39, 1401 (1971).

[11] A. M. MacLeod, K. W. D. Ledingham, and W. T. Morton, Am. J. Phys. 44, 172 (1976).

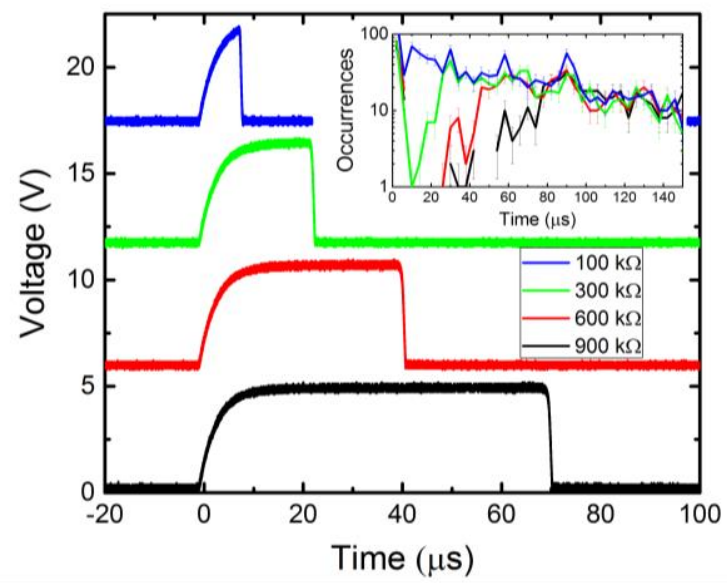

FIG 5. Example oscilloscope traces of the pulses produced by the discriminator for four values of the quenching resistor in series with the LED. From the top, $\mathrm{R}=100$, 300,600 , and $900 \mathrm{k} \Omega$. There is a distribution of pulse widths, and therefore dead times, for pulses produced using the same resistance. Inset: Time between events distribution for the same four resistance values - the increase in the dead time (the time where the data deviate from the exponential trend) due to the pulse width is evident by the deficit of data at short time intervals.

\section{ACKNOWLEDGEMENTS}

Thanks to George Herold of TeachSpin for making the behavior of these LEDs known to the Advanced Lab community through the Advanced Lab Listserv, and to my colleagues Earl Blodgett and Matt Vonk.

[12] M. D. Eisaman, J. Fan, A. Migdall, and S. V. Polyakov, Rev. Sci. Instrum. 82, 071101 (2011).

[13] D. Renker, Nucl. Instrum. Meth. A 567, 48-56 (2006).

[14] V. Savuskan, et al., IEEE Sens. J. 13(6), 2322 - 2328 (2013)

[15] S. Cova, et al., Appl. Opt. 35 (12), 1956-1976 (1996).

[16] S. Cova , et al., J. Mod. Opt. 51(9-10), 1267-1288 (2004)

[17] A. C. Funk and M. Beck, Am. J. Phys. 65, 492 (1997).

[18] P. Koczyk, P. Wiewiór, and C. Radzewicz, Am. J. Phys. 64, 240 (1996).

[19] John R. Taylor, An Introduction to Error Analysis (University Science Books, Sausalito, CA, 1982) p. 207-217.

[20] D. G. Rees, Foundations of Statistics (CRC Press, 1987), p. 163-165.

[21] F.K. Reinhart, J. Appl. Phys. 39 (7), 3426-3434 (1968). 